east England it is commoner on northerly aspects. Again, the conclusion that Hypochoeris radicata is confined to base rich, calcareous soils does not appear to apply to the distribution of this species on the North Downs of Surrey and Kent, where it often indicates the presence of acidic deposits of clay with flints over the chalk.

Grime and Lloyd neglect the consideration of general height of yegetation as a microclimatic determinant for species of small structure. Often this is as important as aspect in controlling the distribution of such species. Could the fact that Medicago lupulina (a prostrate species) is restricted to southerly aspects in the survey simply reflect the preponderance of short turf vegetation on south facing slopes?

This volume contains an abundance of interesting information on the behaviour of grassland species in the Sheffield area. Its application in other areas should be undertaken with considerable care.

Peter D. MoOre

\section{Introducing the Hamiltonian}

Adranced Molecular Quantum Mechanics: An Introduction to Relativistic Quantum Mechanics and the Quantum Theory of Radiation. By R. E. Moss. (Studies in Chemical Physics.) Pp. xvi+ 300. (Chapman and Hall: London; May 1973.) $£ 5.90$.

THE scope of this book is conveyed less accurately by the title than by the subtitle-An Introduction to Relativistic Quantum Mechanics and the Quantum Theory of Radiation. The reader interested in advanced non-relativistic theory should therefore be warned that he will find no discussion of the general area of many-electron quantum mechanics or of theories and techniques for calculating electronic wavefunctions. That being said, Dr Moss is to be congratulated on producing a timely and outstanding monograph in its field.

The book is concerned almost exclusively with the establishment of the many-particle Hamiltonian, including all the "small terms" arising from electron and nuclear spins and internal and applied fields-terms whose effects are nowadays measured with considerable precision by electron and nuclear resonance techniques. The relativistic origin of such terms is imperfectly understood by many of the chemical physicists who take their existence for granted; so also is the quantum field theory needed for a proper treatment of the interaction between a molecule and the radiation field. An acceptable treatment of this area, in the form of a self-contained and up-to-date monograph, was lacking: this text fills the gap admirably.

The reader is assumed to have a know- ledge of elementary non-relativistic quantum mechanics, of which he is reminded in the first ten pages. The next five chapters contain a fairly detailed account of all the matrix algebra, classical mechanics, relativity and electromagnetic theory needed in the second half of the book. Brief but clear explanations often replace a rigorous discussion; but those who find the account a little terse (relativity theory in fourteen pages) will find themselves easily persuaded, and better equipped, to consult the references.

The next part of the book contains an introduction to relativistic wave equations; a good account of the Dirac equation and its reduction to Pauli form; a simplified discussion of the Breit equation and its Pauli form. for a many-electron system; and finally the introduction of nuclei to give the full "molecular Hamiltonian". Unfortunately, the link between this Hamiltonian and the effective or phenomenological Hamiltonian used by spectroscopists receives very little attention. The only application is a chapter on the relativistic theory of the hydrogen atom.

The two remaining chapters contain a simple and well-written introduction to the quantization of the radiation field and its inclusion in the Hamiltonian, leading to the theory of absorption and emission.

Important features are the use of SI units throughout and the high standards of accuracy achieved in reproducing elaborate equations; much checking revealed remarkably few errors or misprints. The quality of production is high and the book deserves to become a standard reference in its field.

\section{R. MCWEENY}

\section{The Natural Balance}

Ecology. By Robert E. Ricklefs. Pp. $\mathrm{x}+861$. (Thomas Nelson: London, September 1973.) £3.95.

THOSE concerned with the conservation of our forests may well be worried by the present plethora of books on the environment and ecology. It is difficult to conceive of a population crash, but one might perhaps hope that competition may slow down the reproductive rate.

Ecology is becoming a word so broad in meaning that it is difficult to guess what a book on this subject might cover. The present work is particularly broad in approach, covering as it does subjects as diverse as perception, time sense, basic properties of the physical environment (these as separate chapters in a part entitled "Ecology of Organisms: Physical Environment"). One aspect which it does not emphasise greatly is the effect of man, pollution, and so forth. The work starts by showing how easily and unknowingly man can upset the environment, but the book is for the biologist. not the environmentalist, and the matter is not laboured.

Apart from the perhaps over-broad approach, the most unusual aspect of the book is the large amount of space devoted to evolutionary aspects of animals and plants. Too many books on ecology tend to ignore the evolutionary aspects of the subject, but this work emphasises them strongly. The author divides the book into eight parts encompassing forty-six chapters, and of these, three parts and sixteen chapters are about evolution in its broadest sense (the parts are called "Natural Selection and Adaptation", "Ecological Genetics and Evolution", "Genetics of Populations"). While I warmly welcome the approach that evolution and ecology are closely interwoven, some of the subject matter in these chapters is again perhaps rather far from the title of the book (for example, a chapter on the rate of evolution).

The other parts cover ecology of organisms, biological environment. ecology of populations and ecology of communities. The first of these three covers predator and prey relationships and competition, as one might expect, but also covers social environments and sexual relationships (including courtship and territory), together with a chapter on the social insects. The part on populations covers population regulation, predator-prey interactions, and evolutionary responses. The part on communiities deals with community ecology, energy flow in ecosystems, relative abundance of species, and species diversity.

The book is clearly written, based around the numerous examples. There is an abundance of clear text figures and photographs, including a particularly pleasing 20-page section of photographs of the vegetative communities of the world. There are nearly 40 pages of references which should enable almost anyone to make a start on the literature on any of the subjects covered. The index is well done, though I should have liked it to include the names of the authors cited. There is relatively little mathematics in most of the book, though it appears in the chapters on population regulation, competition and predatorprey interactions; it should seldom prevent the general reader from understanding the subject.

The general reader will, however, find the work rather long; nevertheless, by selecting those chapters of particular interest he should be able to get a good understanding of the subject. The student wishing to get a broad understanding of the life of whole organisms, especially animals, will find a great deal of interest in this work.

C. M. PERrins 